\title{
Symbolic distance affects two processing loci in the number comparison task
}

\author{
CHRIS ORIET \\ University of Regina, Regina, Saskatchewan, Canada \\ MICHAEL TOMBU \\ York University Centre for Vision Research, Toronto, Ontario, Canada \\ and \\ PIERRE JOLICEUR \\ Université de Montréal, Montréal, Québec, Canada
}

\begin{abstract}
A dual-task procedure was used to investigate the attentional requirements of number processing. The results show that (1) numeric information in Task 2 can be retrieved in parallel with capacity-demanding processing in Task 1 but (2) comparing two quantities requires central capacity, which is depleted by switching from one task to another. This finding resolves an apparent discrepancy in the literature, in which digit magnitude information has not been retrieved in parallel with a second task (Logan \& Schulkind, 2000), despite repeated demonstrations that this information is retrieved autonomously, even when it is deleterious to performance (Henik \& Tzelgov, 1982). A model is proposed to reconcile existing findings with the new ones revealed in the present investigation.
\end{abstract}

In this article, we will investigate the question of whether comparisons of number magnitude require central processing resources. Carrier and Pashler (1995) have argued that retrieval from long-term (episodic) memory cannot be carried out in parallel with response selection in another task, because both operations require access to a single, capacity-limited central process. Logan and Schulkind (2000), however, have argued that retrieval of information from long-term (semantic) memory for responding to one stimulus could be carried out in parallel with retrieval from semantic memory for another stimulus, as long as the same task set was applied to both stimuli. In their Experiment 2, subjects were presented with two digits at varying stimulus onset asynchronies (SOAs) and were asked to perform the same task for each digit (two magnitude judgments or two parity judgments) or different tasks (a magnitude judgment for one digit and a parity judgment for the other). When the task sets applied to the digits were the same, responses to the first stimulus were faster with a match between the categorization of the second stimulus and the categorization of the first stimulus than when the two categorizations did not match. That is, there was crosstalk between the processing of the two digits. When different task sets were applied to the

This work was supported by a Natural Sciences and Engineering Research Council of Canada (NSERC) grant to the third author and by NSERC postgraduate fellowships to the first and second authors. We thank Marg Ingleton for technical assistance. Correspondence concerning this article should be addressed to C. Oriet, Department of Psychology, University of Regina, Regina, SK, S4S 0A2 Canada (e-mail: chris.oriet@ uregina.ca). two stimuli, however, no effect of category compatibility was observed for responses to the first stimulus (i.e., there was no crosstalk between Tasks 1 and 2). Logan and Schulkind argued that number magnitude information was not automatically retrieved from long-term memory in parallel with another task but, rather, was retrieved only when the same task set could be applied to both stimuli.

This conclusion of Logan and Schulkind (2000) seems inconsistent with a large body of research in the area of number processing, which suggests that information about digit magnitude is processed autonomously - that is, whether it is relevant to the task at hand or not. Henik and Tzelgov (1982) found that when subjects were asked to compare the physical size of two digits, determining which digit was physically larger was faster when the physically larger digit was also numerically larger than the digit with which it was compared. In addition, Dehaene and Akhavein (1995) found that more errors were made in judging two stimuli as physically nonidentical when the quantities represented by the two stimuli were equal (e.g., Two and 2) than when they were unequal (e.g., TwO and 4). Furthermore, Windes (1968) showed that the time to report the number of symbols present in a display was shorter when the symbols were, for example, three plus signs than when they were three ones (see also Fox, Shor, \& Steinman, 1971). Taken together, the available evidence strongly suggests that information about a digit's magnitude is accessed whether this information is task relevant or not, and even when it is deleterious to performance. Furthermore, the tasks described above did not require information about number magnitude, but this in- 
formation was retrieved from long-term memory anyway, despite the absence of a task set to process the digit magnitude.

The discrepancy between the results reported by Logan and Schulkind (2000) and those reported in the numberprocessing literature may stem from differences in how the number magnitude information was used in each case. In the performance of the magnitude judgment in Logan and Schulkind's Experiment 2, a direct comparison of two quantities represented in semantic memory was necessary, whereas the information autonomously retrieved from semantic memory in the number-processing experiments described above (Dehaene \& Akhavein, 1995; Henik \& Tzelgov, 1982; Windes, 1968) was merely a source of interference. Possibly, information about number magnitude is accessed whether an appropriate task set is in place or not, but comparing two quantities requires an appropriate task set to be in place before the comparison can be made. Thus, when 5 sevens are presented in a display, subjects might register that there is a difference between the number of digits (five) and the value represented by each of those digits (seven), which interferes with their ability to report the number of digits, rather than the quantity represented by them. It is possible that the fact that a difference is detected is sufficient to cause interference and that no direct comparison is actually computed between five and seven such that the subject would have determined which quantity was larger. This predicts that the size of the interference effect observed in this task would not depend on the difference in the quantities represented by the number and value of the digits, a prediction that is consistent with the results of Tzelgov, Meyer, and Henik (1992).

Evidence for a distinction in the processes underlying access to representations of number magnitude and those underlying comparisons of number magnitude comes from investigation of the symbolic distance effect (Moyer $\&$ Landauer, 1967). The symbolic distance effect in number processing emerges when subjects are asked to compare two quantities. Generally, the time required to determine which member of the pair is larger increases as the numerical difference between the quantities decreases. It is usually assumed that the effect is a consequence of the organization of numbers in semantic space. Many researchers have argued that numbers are represented along a compressed number line (i.e., symbolic distances are greater between smaller numbers than between larger numbers; Banks, Fujii, \& Kayra-Stuart, 1976; Brysbaert, 1995; Rule, 1969). Discrimination of adjacent numbers on the number line is assumed to be more difficult than discrimination of numbers that are further apart, producing the symbolic distance effect. Dehaene and Akhavein (1995) found no symbolic distance effect when subjects were asked to judge whether denotations of different quantities (e.g., EIGHT vs. 2) were physically different but did find that responses were slower when the magnitudes represented by the denotations of the two quantities were equal (e.g., EIGHT vs. 8), providing evidence of access to number magnitude in the absence of a sym- bolic distance effect; similar findings were reported by Tzelgov et al. (1992). Because retrieval of number magnitude information can be demonstrated in the absence of a symbolic distance effect, it is possible that different processes subserve access to number magnitude and comparisons of number magnitude. If so, retrieval of number magnitude information from semantic memory may have occurred autonomously in Logan and Schulkind's (2000) Experiment 2, but the comparison process in Task 2 may have been carried out only when the task sets for Tasks 1 and 2 were the same.

A second explanation for the absence of cross talk between a second digit's magnitude on the processing of an earlier digit in Logan and Schulkind's (2000) Experiment 2 is that information about number magnitude may be retrieved autonomously but comparing numerical quantities can be carried out only when sufficient central resources are available for the comparison process. In their experiment, response times (RTs) were about $275 \mathrm{msec}$ longer in Task 2 when different tasks were performed than when the same tasks were performed in Tasks 1 and 2 . This suggests that there was a large cost of reconfiguring task set when switching from Task 1 to Task 2 . Oriet and Jolicœur (2003) argued that switching between two tasks with stimuli that afford responses in both tasks functions as a hard bottleneck process, preventing even very early processing (such as the processes involved in "cleaning up" a degraded stimulus representation). Possibly, switching between the digit parity and the digit magnitude tasks in Logan and Schulkind's experiment is a process that heavily taxes a limited pool of central resources needed to carry out the comparison of the presented digit and the standard. If so, it might be the case that comparing numerical quantities in Task 2 was not possible when different tasks were performed on the two digits, because switching tasks demanded central resources needed for the comparison process, and not because different task sets were needed for Tasks 1 and 2 .

The attentional requirements of number comparison can be investigated using a dual-task paradigm. If comparing numerical quantities requires central resources, it should not be possible to perform the comparison while central resources are allocated to another task. In the present experiment, we manipulated symbolic distance in Task 2 of a psychological refractory period (PRP) experiment. In PRP experiments, two stimuli are presented at varying stimulus onset asynchronies (SOAs), and each requires a speeded response (see Pashler, 1994, for a review). Typically, RTs in Task 2 increase as SOA decreases, suggesting that some resource needed to complete Task 2 has been allocated to Task 1 and is largely (or completely) unavailable for carrying out Task 2 (e.g., Tombu \& Jolicœur, 2003). Although Task 2 is waiting for resources to be allocated so that its resource-demanding stages can be carried out, stages that precede these bottleneck stages can be carried out in parallel with Task 1 . If these early stages of Task 2 finish before the bottleneck stage of Task 1 is complete, a period of waiting results, in which no further work is done in Task 2 . This pe- 
riod of waiting is sometimes called cognitive slack. Work in Task 2 resumes when the capacity-limited stages in Task 1 have been completed and the limited resource can be allocated to Task 2. Because there is a period of waiting in early Task 2 processing, the duration of an early stage of processing in Task 2 can be increased experimentally without increasing Task 2 RT. In other words, the effect of increasing the difficulty of an early stage of Task 2 is absorbed into cognitive slack (McCann \& Johnston, 1992; Pashler \& Johnston, 1989; Van Selst \& Jolicœur, 1997; see Tombu \& Jolicœur, 2003, for a different model leading to the same prediction). As a result, the PRP paradigm can be used as a diagnostic tool to localize the effect of a manipulated factor: Underadditive interactions with SOA suggest that the factor affected a stage of processing before the bottleneck stage, whereas additive effects with SOA suggest that the factor affected a stage in or after the bottleneck stage.

To determine whether comparing numerical quantities requires central resources, we manipulated symbolic distance in a digit magnitude judgment task as the second task of a PRP experiment. If comparing numerical quantities does not require central resources, we expected to see underadditivity of the symbolic distance effect with decreasing SOA. If comparing numerical quantities does require central resources, we expected to see additivity of this factor with SOA. Task 1 in our experiment was a tone pitch discrimination task, and Task 2 required subjects to decide whether a digit was greater or less than five, so the tasks performed on the stimuli for Tasks 1 and 2 were very different. Switching between very different tasks with univalent stimuli can be accomplished with little or no cost (Allport, Styles, \& Hsieh, 1994; Jersild, 1927; Rogers \& Monsell, 1995; Spector \& Biederman, 1976). Thus, we assume that switching from the tone task to the digit task in our experiment did not produce a large task switch cost and that when central resources were unavailable for Task 2 processing, the lack of availability was due to allocation of these resources to Task 1 processing, rather than to task switching.

The particular tasks used in our dual-task experiment offered us a second means of testing whether digit comparison could be accomplished when central resources were not available. Specifically, we could look for crosstalk from Task 2 onto Task 1, to determine how much of Task 2 has been completed at the time at which a response was selected in Task 1. The logic goes as follows. If the comparison in Task 2 requires central resources, it will be carried out after central processing in Task 1 has finished. Thus, the classification of the digit in Task 2 cannot influence responses in Task 1 (e.g., Hommel, 1998). If, however, the comparison task in Task 2 can be done before central resources become available, the classification of the digit in Task 2 has the potential to influence response selection in Task 1 . The possibility for crosstalk between the tasks, despite the fact that they were different tasks, would emerge from the way in which the tone and the digit were classified. Crosstalk from Task 2 onto Task 1 would be evident if shorter RTs were observed in
Task 1 when the classification of the digit in Task 2 (e.g., higher than five) was compatible with the classification of the tone in Task 1 (high pitch) than if the two classifications were incompatible (higher than five and low pitch).

We also manipulated the compatibility between the classification of the stimuli as greater or less than five and the response to be made for each classification. Dehaene, Bossini, and Giraux (1993) demonstrated that in subjects who read and write from left to right, small quantities are preferentially associated with left responses, whereas large quantities are preferentially associated with right responses (the spatial-numeric associative response compatibility, or SNARC, effect). Therefore, for half of our subjects, less responses were made with the leftmost of the two response keys used in Task 2 (compatible mapping), and for the other half, less responses were made with the rightmost of the two response keys. This manipulation should result in shorter RTs overall for the compatible mapping group than for the incompatible mapping group. Of greater interest, however, was how this variable would interact with other variables in the experiment. For example, it might be the case that digit magnitude information in Task 2 is retrieved in parallel with response selection in Task 1 only when the mapping is compatible. Conceivably, when the mapping is incompatible, more demanding, controlled processing of the digit is carried out than when the mapping is compatible, and digit magnitude information is not retrieved autonomously in Task 2 . Processing of the digit in Task 2 might be deliberately stopped in order to prevent premature execution of the most prepotent response (the one that is most naturally paired with the classification of the Task 2 digit). If this is the case, we would expect to find underadditivity of the symbolic distance effect with decreasing SOA and crosstalk from Task 2 onto Task 1 only for the compatible mapping group. In addition, including this manipulation of response selection difficulty serves as a validity check: If symbolic distance affects a central stage of processing, it should interact with mapping compatibility, and if it does not, these factors should have additive effects.

In summary, Logan and Schulkind (2000) argued that they did not find evidence of retrieval of number magnitude information in Task 2 in parallel with Task 1, because this process cannot be carried out in parallel with a second task unless the task sets for the two tasks are the same. This conclusion is inconsistent with many findings in the number-processing literature, which suggest that number magnitude information is retrieved autonomously whether a task set is in place for processing that information or not. The discrepancy may result from the fact that a comparison between the digit in Task 2 and an internal standard was necessary in Logan and Schulkind's experiment. The present experiment was designed to test two hypotheses for why evidence of parallel processing was not observed in Logan and Schulkind's Experiment 2 . The first hypothesis was that the task sets for Tasks 1 and 2 must be the same for retrieval of number information to be carried out in Task 2 in parallel with Task 1 . 
The second hypothesis was that the retrieval process is autonomous but that the comparison process in Task 2 requires central resources that are unavailable when a difficult task switch is required between Tasks 1 and 2 (i.e., when the stimuli do not uniquely specify the task to be performed, as was the case for the digits in Logan and Schulkind's Experiment 2). Manipulating symbolic distance in the second task of a PRP experiment allows us to determine both whether the task sets for Tasks 1 and 2 must be the same for retrieval of number magnitude information in Task 2 to occur in parallel with Task 1 and whether the comparison process in Task 2 requires central resources. In addition, this paradigm provides us with a means of obtaining converging evidence for the central resource requirements of number comparison by allowing us to check for crosstalk from Task 2 onto Task 1. It also allows us to explore whether a natural mapping of the magnitude of the digit in Task 2 to responses is required for autonomous retrieval of digit magnitude information to occur.

\section{METHOD}

We embedded a number magnitude task as Task 2 in a PRP experiment. Task 1 was a high-/medium-/low-pitch discrimination task. Five levels of SOA were used: 50, 150, 350, 750, and 1,550 msec. In the number magnitude task, the subjects were required to classify a digit as greater than or less than five. The difficulty of the number magnitude task was manipulated by varying the numeric distance of the to-be-judged number from five. The closer a number was to five, the longer it should take to judge that number as less than or greater than five.

\section{Subjects}

One hundred thirteen undergraduate students at the University of Waterloo participated in this experiment in exchange for payment (\$6) or partial fulfillment of course credit. All the subjects had normal or corrected-to-normal vision and normal hearing. The data for 5 subjects were not included, due to low accuracy on one or both of the tasks.

\section{Stimuli and Apparatus}

Each subject was tested individually on a 486 PC. All tones were presented by the internal speaker of the PC. Responses were made using a standard North American QWERTY keyboard.

On each trial, one of three pure tones was presented for $100 \mathrm{msec}$. The pitch of the tone could be 400,1000 , or $2500 \mathrm{~Hz}$, corresponding to the low, medium, and high pitches, respectively. The subjects responded to the low-pitch tone by pressing the "X" key, the mediumpitch tone by pressing the "S" key, and the high-pitch tone by pressing the "Q" key. Responses to the tones were made with the left ring, middle, and index fingers, respectively.

At varying SOAs from the tone, a digit was presented. On every trial, one of eight digits was presented. The digits used were 1,2,3, $4,6,7,8$, and 9 . For half of the subjects, if the digit presented was less than five, the " $<$ " key was pressed, and if the digit was greater than five the adjacent " $>$ " key was pressed. This mapping condition was the compatible response mapping condition, because the "less than" key was associated with stimuli that are less than five (likewise, the "greater than" key was associated with stimuli that are greater than five). For the other half of the subjects, this mapping was reversed (the incompatible mapping condition). No mention of the compatibility relationship was made to the subjects; they were simply told to respond with the " $<$ " and " $>$ " keys. Responses to the digits were made with the right index and middle fingers.

The digits were presented in white on a black background with a height of $1.3^{\circ}$ of visual angle and a width of $0.6^{\circ}$ of visual angle.

\section{Design}

The experiment was divided into six blocks. The first block was a practice block of 60 trials designed to familiarize the subject with the stimulus-response mappings used in the experimental trials. This was followed by five experimental blocks of 120 trials each.

\section{Procedure}

The subjects initiated each trial by pressing the space bar. After $750 \mathrm{msec}$, a tone was presented for $100 \mathrm{msec}$. At varying SOAs, a digit was presented at screen center. The SOAs used were 50, 150, 350,750 , and $1,550 \mathrm{msec}$. The digit stayed on screen until the subject responded. Every combination of tone frequency, digit, and SOA was used equally often over the course of the experiment. After the subject had made both responses, feedback symbols appeared at the center of the screen. Feedback consisted of two symbols presented in locations to the left and to the right of screen center. Each symbol could be either a "+" or a ".$- "$ A "+" in the left position indicated that the tone response on the previous trial was correct, whereas a "-" indicated an error on the previous trial. Likewise, a "+" in the right position indicated a correct response to the digit on the previous trial, whereas a "-" indicated an error on the previous trial. These symbols were cleared from the screen by pressing the space bar, which initiated a new trial.

The subjects were given verbal and written instructions to respond as quickly and accurately as possible in both tasks. They were also instructed to make the tone task their primary task and to always respond to the tone before responding to the digit. In fact, a digit magnitude response would not be accepted until a tone response was made. If, on a given trial, a subject failed to respond to the tone within $1,000 \mathrm{msec}$ of its presentation, a warning message encouraging the subject to respond to the tone more quickly was displayed before the feedback symbols were displayed.

\section{RESULTS AND DISCUSSION}

For each subject, in each cell in which both responses were correct, RTs were screened for outliers, using a modified version of the procedure described by Van Selst and Jolicœur (1994). This procedure is a recursive outlier screening procedure in which the data are checked against a criterion that varies with set size. For more details on the outlier procedure, see Van Selst and Jolicœur, 1994. The outlier procedure was applied separately to the tone RT data and to the digit RT data. If a score on either measure was identified as an outlier, the data for both measures were excluded from analysis. Data from 2.88\% of the trials were discarded because an outlier was identified on the tone response. Of the remaining trials, $2.30 \%$ were discarded because an outlier was identified on the digit response. In total, the outlier analysis led to the exclusion of data from $5.11 \%$ of all the trials from analysis.

For both the tone task and the magnitude judgment task, a mixed model analysis of variance was performed on correct RT data and on accuracy data. There were four within-subjects variables: SOA $(50,150,350,750$, or $1,550 \mathrm{msec}$ ), symbolic distance (near standard or far from standard), tone frequency (low, medium, or high pitch), and digit classification (less than five or more 
Table 1

Mean Tone Response Times (in Milliseconds, With Standard Errors) as a Function of Mapping (Compatible or Incompatible), Symbolic Distance (Near or Far From Standard), Classification of Digit (Less Than Five or More Than Five), Stimulus Onset Asynchrony (SOA; 50, 150, 350, 750, or 1,550 msec), and Tone Pitch (Low, Medium, or High)

\begin{tabular}{|c|c|c|c|c|c|c|c|c|c|}
\hline \multirow[b]{3}{*}{ SOA } & \multirow[b]{3}{*}{ Tone Pitch } & \multicolumn{4}{|c|}{ Near } & \multicolumn{4}{|c|}{ Far } \\
\hline & & \multicolumn{2}{|c|}{ Less } & \multicolumn{2}{|c|}{ More } & \multicolumn{2}{|c|}{ Less } & \multicolumn{2}{|c|}{ More } \\
\hline & & $M$ & $S E$ & $M$ & $S E$ & $M$ & $S E$ & $M$ & $S E$ \\
\hline \multicolumn{10}{|c|}{ Compatible Mapping } \\
\hline \multirow[t]{3}{*}{50} & Low & 663 & 19 & 715 & 25 & 631 & 22 & 698 & 27 \\
\hline & Medium & 680 & 21 & 677 & 20 & 687 & 23 & 704 & 23 \\
\hline & High & 640 & 20 & 633 & 21 & 663 & 22 & 608 & 17 \\
\hline \multirow[t]{3}{*}{150} & Low & 669 & 22 & 654 & 22 & 650 & 17 & 671 & 24 \\
\hline & Medium & 679 & 24 & 669 & 20 & 694 & 25 & 680 & 23 \\
\hline & High & 617 & 20 & 615 & 18 & 628 & 21 & 601 & 19 \\
\hline \multirow[t]{3}{*}{350} & Low & 638 & 18 & 640 & 19 & 641 & 18 & 627 & 21 \\
\hline & Medium & 637 & 21 & 650 & 21 & 671 & 21 & 662 & 25 \\
\hline & High & 574 & 22 & 586 & 15 & 582 & 17 & 576 & 15 \\
\hline \multirow[t]{3}{*}{750} & Low & 643 & 19 & 634 & 19 & 620 & 18 & 614 & 17 \\
\hline & Medium & 653 & 23 & 678 & 21 & 633 & 19 & 679 & 20 \\
\hline & High & 594 & 18 & 578 & 20 & 580 & 15 & 590 & 20 \\
\hline \multirow[t]{3}{*}{1,550} & Low & 632 & 21 & 640 & 23 & 650 & 21 & 636 & 18 \\
\hline & Medium & 668 & 23 & 654 & 18 & 650 & 18 & 661 & 21 \\
\hline & High & 604 & 19 & 593 & 22 & 597 & 19 & 590 & 20 \\
\hline \multicolumn{10}{|c|}{ Incompatible Mapping } \\
\hline \multirow[t]{3}{*}{50} & Low & 674 & 19 & 678 & 25 & 682 & 22 & 719 & 27 \\
\hline & Medium & 673 & 21 & 689 & 20 & 758 & 23 & 707 & 23 \\
\hline & High & 657 & 20 & 658 & 21 & 666 & 22 & 636 & 17 \\
\hline \multirow[t]{3}{*}{150} & Low & 676 & 22 & 677 & 22 & 662 & 17 & 694 & 24 \\
\hline & Medium & 714 & 24 & 691 & 20 & 727 & 25 & 713 & 23 \\
\hline & High & 635 & 20 & 628 & 18 & 646 & 21 & 601 & 19 \\
\hline \multirow[t]{3}{*}{350} & Low & 639 & 18 & 656 & 19 & 647 & 18 & 667 & 21 \\
\hline & Medium & 678 & 21 & 679 & 21 & 642 & 21 & 687 & 25 \\
\hline & High & 636 & 22 & 575 & 15 & 617 & 17 & 597 & 15 \\
\hline \multirow[t]{3}{*}{750} & Low & 646 & 19 & 650 & 19 & 642 & 18 & 625 & 17 \\
\hline & Medium & 666 & 23 & 694 & 21 & 686 & 19 & 663 & 20 \\
\hline & High & 607 & 18 & 602 & 20 & 592 & 15 & 626 & 20 \\
\hline \multirow[t]{3}{*}{1,550} & Low & 645 & 21 & 683 & 23 & 662 & 21 & 655 & 18 \\
\hline & Medium & 685 & 23 & 682 & 18 & 656 & 18 & 703 & 21 \\
\hline & High & 619 & 19 & 645 & 22 & 614 & 19 & 607 & 20 \\
\hline
\end{tabular}

than five). By including tone frequency and digit classification as factors in the analysis, we were able to look for crosstalk between Tasks 1 and 2. The digits 3, 4, 6, and 7 were defined as being close in symbolic distance space, whereas the digits $1,2,8$, and 9 were defined as being far in symbolic distance space. The stimulusresponse mapping for the magnitude judgment task was manipulated as a between-subjects factor with two levels (compatible and incompatible mapping). Mean RTs for the tone and the digit tasks are listed in Tables 1 and 2, respectively. The mean proportions of correct responses in the tone and the digit tasks are listed in Tables 3 and 4, respectively.

The effect of symbolic distance on Task 2 RTs was underadditive with decreasing SOA, with a residual effect persisting at the shortest SOAs, leading to a significant SOA $\times$ symbolic distance interaction $[F(4,424)=4.16$, $M S_{\mathrm{e}}=13,009.3, p<.003$; see Figure 1]. Reducing SOA from 1,550 to $350 \mathrm{msec}$ resulted in a reduction in the magnitude of the effect, but further reducing SOA resulted in no additional reduction. This finding was sup- ported by the outcome of a separate analysis in which only the first three levels of the SOA variable, symbolic distance, and response mapping were included as factors. This analysis showed that the effect of symbolic distance was significant when averaged over these three SOAs $\left[F(1,106)=36.7, M S_{\mathrm{e}}=3,467.3, p<.0001\right.$, for the main effect of symbolic distance], that the effect did not vary as a function of SOA $\left[F(2,212)=0.09, M S_{\mathrm{e}}=\right.$ $7,771.9, p>.91$, for the symbolic distance $\times$ SOA interaction], and that there was no three-way interaction between symbolic distance, SOA, and response mapping $\left[F(2,212)=1.16, M S_{\mathrm{e}}=7,771.9, p>.31\right]$. These analyses show that the symbolic distance effect was not completely absorbed into cognitive slack, which suggests that symbolic distance has its effect at both an early and a central (or late) stage of processing; at least part of the comparison process for the digit in Task 2 was carried out in parallel with Task 1. Furthermore, this evidence of retrieval of number magnitude information from semantic memory was observed despite the fact that different task sets were required for Tasks 1 and 2 . 
Table 2

Mean Digit Response Times (in Milliseconds, With Standard Errors) as a Function of Mapping (Compatible or Incompatible), Symbolic Distance (Near or Far From Standard), Classification of Digit (Less Than Five or More Than Five), Stimulus Onset Asynchrony (SOA; 50, 150, 350, 750, or 1,550 msec), and Tone Pitch (Low, Medium, or High)

\begin{tabular}{|c|c|c|c|c|c|c|c|c|c|}
\hline \multirow[b]{3}{*}{$\underline{\text { SOA }}$} & \multirow[b]{3}{*}{ Tone Pitch } & \multicolumn{4}{|c|}{ Near } & \multicolumn{4}{|c|}{ Far } \\
\hline & & \multicolumn{2}{|c|}{ Less } & \multicolumn{2}{|c|}{ More } & \multicolumn{2}{|c|}{ Less } & \multicolumn{2}{|c|}{ More } \\
\hline & & $M$ & $S E$ & $M$ & $S E$ & $M$ & $S E$ & $M$ & $S E$ \\
\hline \multicolumn{10}{|c|}{ Compatible Mapping } \\
\hline \multirow[t]{3}{*}{50} & Low & 1,052 & 32.1 & 1,132 & 38.1 & 964 & 36.8 & 1,078 & 37.2 \\
\hline & Medium & 1,086 & 32.8 & 1,077 & 33.2 & 1,033 & 32.2 & 1,066 & 35.5 \\
\hline & High & 1,042 & 32.2 & 1,046 & 33.7 & 1,045 & 35.7 & 976 & 31.4 \\
\hline \multirow[t]{3}{*}{150} & Low & 972 & 36.9 & 977 & 34.1 & 890 & 29.2 & 972 & 39.4 \\
\hline & Medium & 979 & 36.9 & 973 & 31.4 & 945 & 34.6 & 950 & 35.0 \\
\hline & High & 916 & 34.8 & 928 & 32.0 & 900 & 32.9 & 872 & 30.6 \\
\hline \multirow[t]{3}{*}{350} & Low & 747 & 30.9 & 774 & 32.2 & 719 & 28.8 & 742 & 30.9 \\
\hline & Medium & 759 & 30.8 & 792 & 30.7 & 744 & 31.9 & 763 & 34.8 \\
\hline & High & 713 & 33.8 & 741 & 26.3 & 686 & 26.2 & 693 & 27.9 \\
\hline \multirow[t]{3}{*}{750} & Low & 594 & 22.1 & 600 & 19.7 & 528 & 20.3 & 546 & 17.6 \\
\hline & Medium & 610 & 25.7 & 625 & 25.5 & 533 & 19.7 & 588 & 21.8 \\
\hline & High & 588 & 23.2 & 601 & 23.1 & 534 & 17.8 & 542 & 20.3 \\
\hline \multirow[t]{3}{*}{1,550} & Low & 553 & 19.9 & 550 & 18.3 & 503 & 13.8 & 517 & 13.7 \\
\hline & Medium & 553 & 20.4 & 554 & 19.1 & 499 & 13.8 & 506 & 14.6 \\
\hline & High & 560 & 16.3 & 561 & 18.2 & 493 & 15.9 & 511 & 15.7 \\
\hline \multicolumn{10}{|c|}{ Incompatible Mapping } \\
\hline \multirow[t]{3}{*}{50} & Low & 1,165 & 32 & 1,137 & 38 & 1,145 & 37 & 1,157 & 37 \\
\hline & Medium & 1,167 & 33 & 1,144 & 33 & 1,214 & 32 & 1,145 & 36 \\
\hline & High & 1,129 & 32 & 1,144 & 34 & 1,121 & 36 & 1,093 & 31 \\
\hline \multirow[t]{3}{*}{150} & Low & 1,058 & 37 & 1,027 & 34 & 1,000 & 29 & 1,047 & 39 \\
\hline & Medium & 1,124 & 37 & 1,050 & 31 & 1,100 & 35 & 1,049 & 35 \\
\hline & High & 1,028 & 35 & 998 & 32 & 1,006 & 33 & 935 & 30 \\
\hline \multirow[t]{3}{*}{350} & Low & 858 & 31 & 846 & 32 & 841 & 29 & 835 & 31 \\
\hline & Medium & 870 & 31 & 853 & 31 & 838 & 32 & 844 & 35 \\
\hline & High & 855 & 34 & 770 & 26 & 790 & 26 & 780 & 28 \\
\hline \multirow[t]{3}{*}{750} & Low & 689 & 22 & 652 & 20 & 645 & 20 & 623 & 18 \\
\hline & Medium & 702 & 26 & 686 & 26 & 647 & 20 & 642 & 22 \\
\hline & High & 690 & 23 & 671 & 23 & 620 & 18 & 648 & 20 \\
\hline \multirow[t]{3}{*}{1,550} & Low & 652 & 20 & 620 & 18 & 586 & 14 & 574 & 14 \\
\hline & Medium & 643 & 20 & 613 & 19 & 579 & 14 & 583 & 15 \\
\hline & High & 625 & 16 & 626 & 18 & 593 & 16 & 586 & 16 \\
\hline
\end{tabular}

Partial underadditivity of the symbolic distance effect was observed whether the stimulus-response mapping of digit classification was spatially compatible or incompatible. In our results, this SNARC effect was additive with SOA for Task 2 RTs $\left[F(4,424)=0.40, M S_{\mathrm{e}}=\right.$ $56,778.0, p>.80]$, suggesting that it affects a central stage of processing in Task 2 (see Figure 2). Consistent with this interpretation, the SNARC effect interacted with the symbolic distance effect $\left[F(1,106)=6.94, M S_{\mathrm{e}}=\right.$ $16,072.4, p<.01]$, which we have already argued has a central (and an early) locus. Interestingly, the symbolic distance effect was underadditive with the SNARC effect (i.e., the effect was smaller on both RT and accuracy with slower, less accurate, incompatible responses than with faster, more accurate, compatible responses). One interpretation of this is that some aspect of resolving the difficulty of an incompatible mapping could be carried out in parallel with some aspect of resolving the difficulty of classifying digits symbolically proximal to an internal standard of comparison, but this process could not be carried out in parallel with the capacity-demanding stages of Task 1 .
With the exception of the classification of the digit as less than five or more than five, all of the main effects were significant at $p<.006$. However, these main effects were qualified by a significant three-way interaction and two significant four-way interactions. SOA, tone frequency, and the classification of the digit as greater than or less than five interacted in the following way $[F(8,848)=$ $\left.4.39, M S_{\mathrm{e}}=12,152.2, p<.001\right]$. Classifying the digit as greater than five was faster when the digit followed a high-pitch tone than when it followed a low-pitch tone, and classifying the digit as less than five was faster when the digit followed a low-pitch tone than when it followed a high-pitch tone. This effect increased as SOA decreased. For the medium tone, RTs did not vary systematically with digit classification and tended to be longer than for either high or low tones. This suggests that the classification of the tone as high or low primed the classification of the digit as more or less than five, having more of an effect as the overlap between the tasks increased, perhaps by activating the responses associated with the digit, prior to response selection in Task 2 (Hommel, 1998). Responses following medium tones, in contrast, were 
Table 3

Mean Proportion of Correct Tone Responses (With Standard Errors) as a Function of Mapping (Compatible or Incompatible), Symbolic Distance (Near or Far From Standard), Classification of Digit (Less Than Five or More Than Five), Stimulus Onset Asynchrony (SOA; 50, 150, 350, 750, or 1,550 msec), and Tone Pitch (Low, Medium, or High)

\begin{tabular}{|c|c|c|c|c|c|c|c|c|c|}
\hline \multirow[b]{3}{*}{ SOA } & \multirow[b]{3}{*}{ Tone Pitch } & \multicolumn{4}{|c|}{ Near } & \multicolumn{4}{|c|}{ Far } \\
\hline & & \multicolumn{2}{|c|}{ Less } & \multicolumn{2}{|c|}{ More } & \multicolumn{2}{|c|}{ Less } & \multicolumn{2}{|c|}{ More } \\
\hline & & $M$ & $\overline{S E}$ & $M$ & $\overline{S E}$ & $M$ & $\overline{S E}$ & $M$ & $\overline{S E}$ \\
\hline \multicolumn{10}{|c|}{ Compatible Mapping } \\
\hline \multirow[t]{3}{*}{50} & Low & .92 & .01 & .86 & .02 & .93 & .02 & .89 & .02 \\
\hline & Medium & .94 & .01 & .94 & .02 & .90 & .02 & .90 & .01 \\
\hline & High & .94 & .01 & .95 & .01 & .94 & .02 & .96 & .01 \\
\hline \multirow[t]{3}{*}{150} & Low & .91 & .01 & .90 & .02 & .93 & .01 & .89 & .02 \\
\hline & Medium & .94 & .01 & .94 & .01 & .94 & .01 & .94 & .01 \\
\hline & High & .95 & .01 & .95 & .01 & .95 & .01 & .96 & .01 \\
\hline \multirow[t]{3}{*}{350} & Low & .91 & .02 & .94 & .01 & .93 & .02 & .93 & .01 \\
\hline & Medium & .97 & .01 & .96 & .01 & .93 & .01 & .96 & .01 \\
\hline & High & .97 & .01 & .96 & .01 & .96 & .01 & .97 & .01 \\
\hline \multirow[t]{3}{*}{750} & Low & .90 & .02 & .92 & .02 & .93 & .01 & .93 & .01 \\
\hline & Medium & .93 & .01 & .96 & .02 & .96 & .02 & .95 & .01 \\
\hline & High & .96 & .01 & .96 & .01 & .97 & .01 & .95 & .01 \\
\hline \multirow{3}{*}{1,550} & Low & .91 & .01 & .90 & .02 & .94 & .01 & .91 & .02 \\
\hline & Medium & .96 & .01 & .95 & .01 & .95 & .01 & .93 & .01 \\
\hline & High & .98 & .01 & .95 & .01 & .97 & .01 & .96 & .01 \\
\hline \multicolumn{10}{|c|}{ Incompatible Mapping } \\
\hline \multirow[t]{3}{*}{50} & Low & .93 & .01 & .90 & .02 & .93 & .02 & .89 & .02 \\
\hline & Medium & .95 & .01 & .95 & .02 & .89 & .02 & .92 & .01 \\
\hline & High & .93 & .01 & .95 & .01 & .93 & .02 & .94 & .01 \\
\hline \multirow[t]{3}{*}{150} & Low & .92 & .01 & .90 & .02 & .94 & .01 & .89 & .02 \\
\hline & Medium & .95 & .01 & .93 & .01 & .91 & .01 & .91 & .01 \\
\hline & High & .94 & .01 & .96 & .01 & .94 & .01 & .97 & .01 \\
\hline \multirow[t]{3}{*}{350} & Low & .94 & .02 & .93 & .01 & .93 & .02 & .93 & .01 \\
\hline & Medium & .93 & .01 & .95 & .01 & .92 & .01 & .94 & .01 \\
\hline & High & .96 & .01 & .97 & .01 & .97 & .01 & .97 & .01 \\
\hline \multirow[t]{3}{*}{750} & Low & .90 & .02 & .91 & .02 & .93 & .01 & .92 & .01 \\
\hline & Medium & .93 & .01 & .92 & .02 & .93 & .02 & .92 & .01 \\
\hline & High & .97 & .01 & .98 & .01 & .98 & .01 & .97 & .01 \\
\hline \multirow[t]{3}{*}{1,550} & Low & .93 & .01 & .91 & .02 & .92 & .01 & .91 & .02 \\
\hline & Medium & .93 & .01 & .93 & .01 & .94 & .01 & .93 & .01 \\
\hline & High & .96 & .01 & .98 & .01 & .97 & .01 & .97 & .01 \\
\hline
\end{tabular}

not primed, which is not surprising given that there is no reason to expect that the medium tone pitch would be conceptually related to classifying a digit as more or less than five. This three-way interaction was qualified by interactions with symbolic distance $[F(8,848)=2.13$, $\left.M S_{\mathrm{e}}=13,328.8, p<.04\right]$ and response mapping $\left[F(8,848)=2.40, M S_{\mathrm{e}}=12,152.2, p<.02\right]$. The interaction with symbolic distance revealed that the pattern described above was more pronounced for digits that were symbolically far from the standard, with any crosstalk at all constrained to the shortest SOA. We will defer further discussion of this important finding for later in this article. The interaction with response mapping suggests that the three-way interaction described above was more pronounced for the compatible mapping group than for the incompatible mapping group, perhaps suggesting that when response selection is made more difficult (and is, therefore, more controlled), it is less susceptible to extraneous influences, such as priming from the tone.

The accuracy of classifying the digit was very high overall, ranging from $85 \%$ to $99 \%$ across conditions. As with the RT data, all of the main effects (except for the classification of the digit as either less than five or more than five) were significant, as were several two-way interactions. These were qualified by three-way interactions, all but one of which was qualified by a four-way interaction. The classification of the digit as less or more than five depended on the pitch of the tone. Accuracy was somewhat higher for high-tone/more and low-tone/ less pairings than for the opposite pairings, with this pattern more pronounced in the response-compatible mapping group than in the response-incompatible mapping group $\left[F(2,212)=5.18, M S_{\mathrm{e}}=0.01, p<.007\right]$. A significant four-way interaction between response mapping, SOA, digit classification as less or more than five, and symbolic distance was not interpretable, because there is no a priori reason to expect the digit classification variable to participate in interactions that do not include tone frequency as a factor. There was, however, a significant four-way interaction between tone frequency, SOA, digit classification as less or more than five, and symbolic distance $\left[F(8,848)=2.46, M S_{\mathrm{e}}=0.006, p<\right.$ $.02]$. This interaction roughly mirrored the pattern observed in RTs. Responses to digits that were greater than 
Table 4

Mean Proportion of Correct Digit Responses (With Standard Errors) as a Function of Mapping (Compatible or Incompatible), Symbolic Distance (Near or Far From Standard), Classification of Digit (Less Than Five or More Than Five), Stimulus Onset Asynchrony (SOA; 50, 150, 350, 750, or 1,550 msec), and Tone Pitch (Low, Medium, or High)

\begin{tabular}{|c|c|c|c|c|c|c|c|c|c|}
\hline \multirow[b]{3}{*}{$\underline{\text { SOA }}$} & \multirow[b]{3}{*}{ Tone Pitch } & \multicolumn{4}{|c|}{ Near } & \multicolumn{4}{|c|}{ Far } \\
\hline & & \multicolumn{2}{|c|}{ Less } & \multicolumn{2}{|c|}{ More } & \multicolumn{2}{|c|}{ Less } & \multicolumn{2}{|c|}{ More } \\
\hline & & $M$ & $S E$ & $M$ & $S E$ & $M$ & $S E$ & $M$ & $S E$ \\
\hline \multicolumn{10}{|c|}{ Compatible Mapping } \\
\hline \multirow[t]{3}{*}{50} & Low & .95 & .01 & .85 & .02 & .98 & .01 & .93 & .01 \\
\hline & Medium & .94 & .02 & .93 & .01 & .99 & .01 & .96 & .01 \\
\hline & High & .94 & .01 & .91 & .02 & .95 & .01 & .97 & .01 \\
\hline \multirow[t]{3}{*}{150} & Low & .95 & .01 & .87 & .02 & .98 & .01 & .96 & .01 \\
\hline & Medium & .94 & .02 & .92 & .01 & .97 & .01 & .96 & .01 \\
\hline & High & .93 & .01 & .93 & .01 & .98 & .01 & .96 & .01 \\
\hline \multirow[t]{3}{*}{350} & Low & .93 & .01 & .92 & .01 & .99 & .01 & .95 & .01 \\
\hline & Medium & .95 & .01 & .92 & .01 & .97 & .01 & .96 & .01 \\
\hline & High & .95 & .01 & .93 & .01 & .97 & .01 & .96 & .01 \\
\hline \multirow[t]{3}{*}{750} & Low & .94 & .01 & .93 & .01 & .99 & .01 & .97 & .01 \\
\hline & Medium & .94 & .01 & .93 & .01 & .99 & .01 & .98 & .01 \\
\hline & High & .92 & .01 & .96 & .01 & .98 & .01 & .98 & .01 \\
\hline \multirow{3}{*}{1,550} & Low & .95 & .01 & .96 & .01 & .99 & .01 & .98 & .01 \\
\hline & Medium & .94 & .01 & .97 & .01 & .98 & .01 & .99 & .01 \\
\hline & High & .95 & .01 & .96 & .01 & .99 & .01 & .98 & .01 \\
\hline \multicolumn{10}{|c|}{ Incompatible Mapping } \\
\hline \multirow[t]{3}{*}{50} & Low & .91 & .01 & .94 & .02 & .91 & .01 & .92 & .01 \\
\hline & Medium & .87 & .02 & .95 & .01 & .93 & .01 & .95 & .01 \\
\hline & High & .92 & .01 & .93 & .02 & .93 & .01 & .96 & .01 \\
\hline \multirow[t]{3}{*}{150} & Low & .88 & .01 & .91 & .02 & .90 & .01 & .94 & .01 \\
\hline & Medium & .87 & .02 & .95 & .01 & .94 & .01 & .97 & .01 \\
\hline & High & .91 & .01 & .93 & .01 & .94 & .01 & .94 & .01 \\
\hline \multirow[t]{3}{*}{350} & Low & .90 & .01 & .95 & .01 & .92 & .01 & .95 & .01 \\
\hline & Medium & .91 & .01 & .94 & .01 & .95 & .01 & .98 & .01 \\
\hline & High & .93 & .01 & .94 & .01 & .97 & .01 & .97 & .01 \\
\hline \multirow[t]{3}{*}{750} & Low & .95 & .01 & .93 & .01 & .96 & .01 & .97 & .01 \\
\hline & Medium & .92 & .01 & .94 & .01 & .96 & .01 & .96 & .01 \\
\hline & High & .95 & .01 & .95 & .01 & .95 & .01 & .98 & .01 \\
\hline \multirow[t]{3}{*}{1,550} & Low & .94 & .01 & .95 & .01 & .95 & .01 & .97 & .01 \\
\hline & Medium & .94 & .01 & .95 & .01 & .97 & .01 & .97 & .01 \\
\hline & High & .94 & .01 & .96 & .01 & .96 & .01 & .97 & .01 \\
\hline
\end{tabular}

five were more accurate following a high tone than following a low tone, and responses to digits that were less than five were more accurate following a low tone than following a high tone. This pattern was constrained to the shortest SOAs and was more pronounced with digits that were symbolically far from the standard than with digits that were symbolically near the standard.

We considered two hypotheses with which to explain Logan and Schulkind's (2000) finding that there was no crosstalk between Tasks 1 and 2 in their Experiment 2. The first hypothesis was that retrieval of number magnitude information can proceed in parallel with another task but comparing the retrieved quantity with an internal standard of comparison can be carried only out when the same task set is in place for Tasks 1 and 2. The second hypothesis was that retrieval of number magnitude information can proceed in parallel with another task but the comparison process requires central resources, which were also needed to complete the task switch from Task 1 to Task 2 in Logan and Schulkind's experiment. The effect of not having central resources available for the comparison process might be that this process is delayed until the task switch is complete. In our experiment, the subjects switched between discriminating the pitch of a tone and classifying a digit relative to a fixed standard. Possibly, switching between such dissimilar tasks can be accomplished with little requirement for central resources.

To test the first of these hypotheses, we looked for evidence of number magnitude retrieval in Task 2 while a different task was carried out in Task 1. In our experiment, the subjects were required to make a speeded tone pitch discrimination in Task 1 and a judgment of digit magnitude in Task 2. These tasks require different task sets. Nevertheless, information about the classification of the digit was retrieved in parallel with a demanding stage of Task 1 (perhaps response selection), despite the fact that different task sets were required for Tasks 1 and 2. Additional support for this assertion comes from analysis of Task 1 RTs. Only two of the five main effects were significant in this analysis (tone frequency and SOA), and each of these main effects was qualified by significant two-way interactions, which were, in turn, qualified by higher order interactions, and so will not be described further. As for Task 2 RTs, the three-way interaction be- 


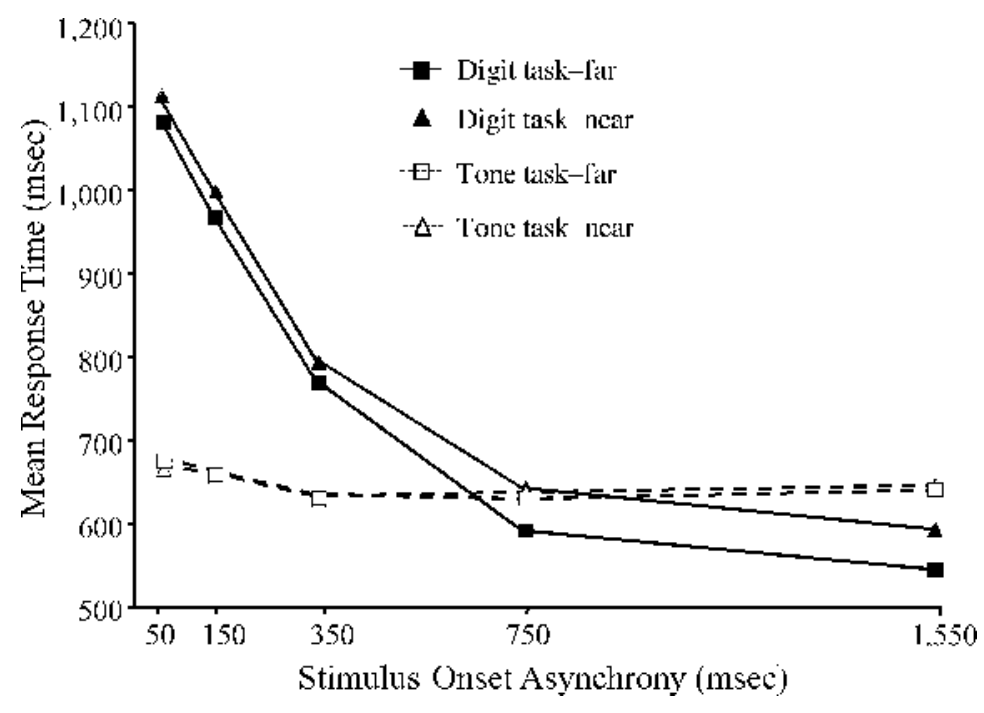

Figure 1. Mean response times (RTs) for the digit task (RT2) and the tone task (RT1) as a function of stimulus onset asynchrony and symbolic distance. Squares, far digits; triangles, near digits; open symbols/dotted lines, Task 1 RTs; closed symbols/solid lines, Task 2 RTs.

tween SOA, tone frequency, and the classification of the digit as less or more than five was significant $[F(8,848)=$ 3.64, $\left.M S_{\mathrm{e}}=8,306.5, p<.001\right]$. As for Task 2 RTs, this interaction resulted from crosstalk between Tasks 1 and 2. Specifically, responses to the tone were made more quickly with high-tone/more-than-five and low-tone/lessthan-five pairings than with the opposite pairings. This effect became more pronounced as the overlap between the tasks increased (i.e., as SOA decreased), indicating that the magnitude of the digit in Task 2 influenced the speed of classifying the tone in Task 1 . This suggests that information about the magnitude of the digit in Task 2 was retrieved in parallel with a central stage of processing in Task 1, despite the fact that different tasks were performed in Task 1 and Task 2 . Interestingly, because the subjects were instructed to classify the digit as greater than five or less than five, rather than as high or low, the classification of the stimuli in this experiment must have been rather abstract for compatibility effects to be observed between the digit and the tone. That is, the tone-

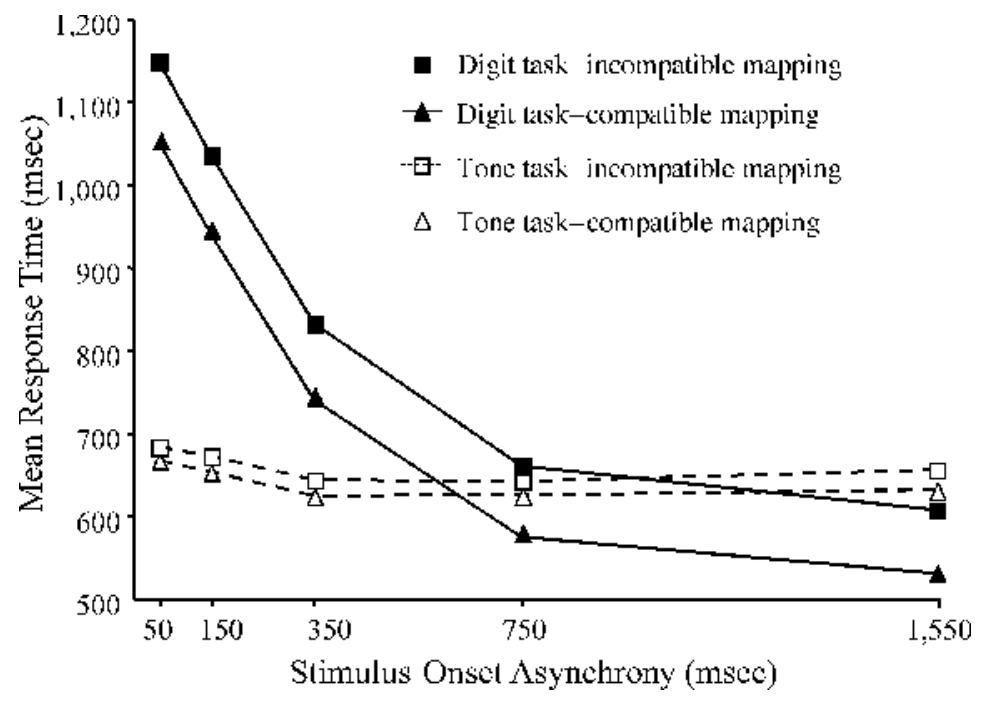

Figure 2. Mean response times (RTs) for the digit task (RT2) and the tone task (RT1) as a function of stimulus onset asynchrony and symbolic distance. Squares, incompatible response mapping; triangles, compatible response mapping; open symbols/dotted lines, Task 1 RTs; closed symbols/solid lines, Task 2 RTs. 
digit compatibility must be at the level of the meaning of the stimuli, rather than at the level of the verbal labels for the responses suggested by the instructions. In any case, the tone-digit compatibility effects cannot be attributed to a match at the level of the overt response, because different sets of response keys were used in the two tasks. The three-way interaction between SOA, tone frequency, and classification of the digit as less or more than five was qualified by two four-way interactions, one with symbolic distance $\left[F(8,848)=3.47, M S_{\mathrm{e}}=7,979.0, p<\right.$ $.001]$ and one with response mapping $[F(8,848)=2.50$, $\left.M S_{\mathrm{e}}=8,306.5, p<.02\right]$. This is precisely what was observed for Task 2 RTs, and the patterns of data are quite similar between Task 2 and Task 1 RTs. The three-way interaction described above was much more pronounced for digits that were symbolically far from the standard than for digits that were symbolically near the standard (see Figure 3). Discussion of this important finding (and the corroborating finding in Task 2 RTs) will be deferred to a later point in the article. The three-way interaction between SOA, tone frequency, and classification of the digit as less or more than five was more pronounced in the compatible response mapping group than in the incompatible response mapping group. One interpretation of this finding is that subjects in the incompatible group were able to actively suppress the information retrieved about the digit because automatic activation of the response naturally associated with, for example, the response less than five, would lead to automatic activation of the wrong response (the left response, which is mapped to more than five in this group). Because this information was suppressed in Task 2, it had minimal impact on classifying the pitch of the tone in Task 1.

For accuracy, the main effects of tone frequency and SOA were significant, but these main effects were qualified by a three-way interaction. Responses tended to be more accurate with tone/digit correspondence (low-tone/ less-than-five digit classification or high-tone/more-thanfive digit classification) than with tone/digit noncorrespondence (low-tone/more-than-five digit classification or high-tone/less-than-five digit classification), and these effects increased with decreasing SOA, leading to a significant SOA $\times$ tone frequency $\times$ digit classification interaction $\left[F(8,848)=3.39, M S_{\mathrm{e}}=0.006, p<.001\right]$. This result parallels that found for Task 1 response times, and indicates that the classification of the digit in Task 2 influenced the accuracy of response selection in Task 1, providing further evidence that digit magnitude information in Task 2 was retrieved in parallel with response selection in Task 1.

The results suggest that it is not necessary to apply the same task set to both tasks in order for information about digit magnitude to be retrieved in Task 2, in parallel with Task 1. Rather, it is sufficient that the task sets for the two tasks are not mutually incompatible - that is, that the two task sets can be held concurrently. Sudevan and Tay-

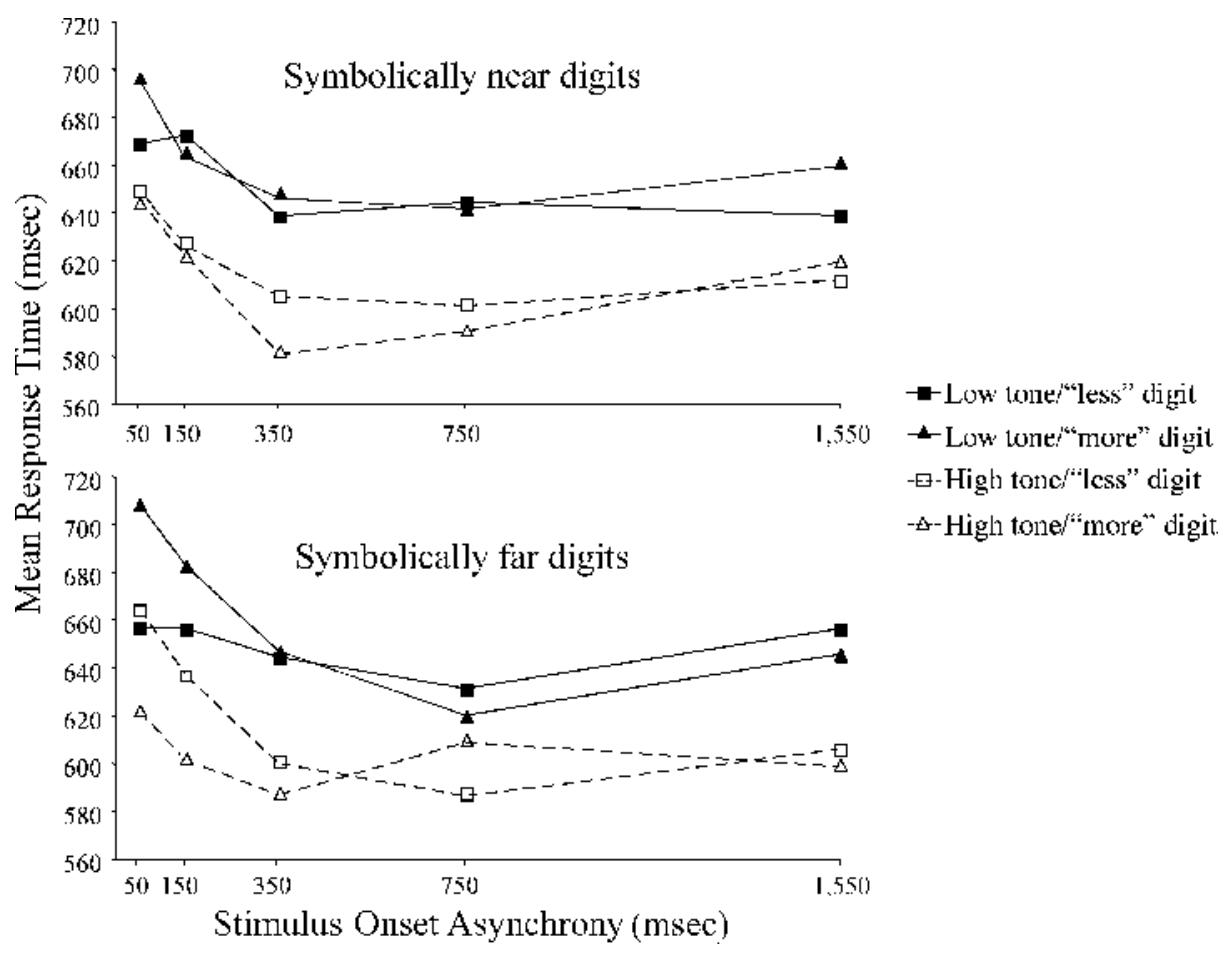

Figure 3. Mean response times for the tone task (Task 1) as a function of compatibility with digit classification in Task 2 and symbolic distance. Top panel: near digits. Bottom panel: far digits. Squares, digits classified as less than five; triangles, digits classified as more than five; open symbols/ dotted lines, high-pitch tones; closed symbols/solid lines, low-pitch tones. 
lor (1987) suggested that a task set for processing digit magnitude could not be held active concurrently with a task set for processing digit parity. However, it is possible that a task set for processing digit magnitude can be held concurrently with a task set for processing the pitch of a tone. It should be noted that Carrier and Pashler (1995) found no evidence that information was retrieved from long-term memory in parallel with demanding stages of a second task. This would seem to contradict our findings, given that in their experiment, task sets for the first task (a tone pitch discrimination task) and the second task (long-term memory retrieval) could likely have been held concurrently. We suspect that the critical difference between their study and the present work is that information was retrieved from episodic memory in their task, whereas information was retrieved from semantic memory in ours. Possibly, retrieving information from episodic memory requires central resources, but retrieving information from semantic memory (digit magnitude, in the present study) does not.

The second hypothesis was that switching from the parity task to the digit magnitude task in Logan and Schulkind's (2000) study may be a process that requires access to a limited pool of central resources, resources that are also needed to compare numerical quantities. Thus, the second hypothesis was that a lack of availability of central resources during task switching prevented Logan and Schulkind from finding evidence of retrieval of digit magnitude information in parallel with another task, and not the absence of a task set for processing that information per se. In our experiment, Tasks 1 and 2 were highly dissimilar (discriminating the pitch of a tone and classifying a digit relative to a fixed standard). Previous research (Allport et al., 1994; Jersild, 1927; Rogers \& Monsell, 1995; Spector \& Biederman, 1976) suggests that switching between very different tasks can be accomplished with little or no cost, which further suggests that such task switches have little requirement for central resources. The findings of partial underadditivity of the symbolic distance effect and crosstalk from Task 2 to Task 1 suggest that a lack of central resources due to switching between tasks was the reason that crosstalk from Task 2 to Task 1 was not observed in Logan and Schulkind's Experiment 2. When central resources are not limited by a difficult task switch (as in the present experiment) and can be allocated to Task 2 in parallel with Task 1 (albeit, at a cost to Task 1 performance, as will be described below), retrieval of digit magnitude information is carried out in parallel with the central stages of a second task, even when this task is very different from the first.

Additional evidence supporting the claim that resources were simultaneously allocated to Task 1 and Task 2 comes from analysis of Task 1 RTs. According to the central capacity sharing (CCS) model of dual-task performance (Tombu \& Jolicœur, 2003), when processing capacity is shared between two tasks in the PRP paradigm, RTs in the first task are predicted to increase as SOA is decreased (see also Navon \& Miller, 2002). The CCS model makes the same predictions for Task 2 RTs as the all-ornone bottleneck model (Pashler, 1994). Therefore, evidence for capacity sharing does not invalidate inferences based on Task 2 RTs. The predictions for Task 1 RTs are, however, different between the CCS and the all-or-none bottleneck models. Specifically, the CCS model predicts an increase in Task 1 RTs as SOA is decreased, when capacity is shared between Tasks 1 and 2; the all-or-none bottleneck model predicts that Task 1 RTs should not vary as a function of SOA. Analysis of Task 1 RTs in our experiment revealed a reliable increase in RTs (about $40 \mathrm{msec}$ ) as SOA was decreased over the range of SOAs tested $\left[F(4,424)=27.5, M S_{\mathrm{e}}=16,083.5, p<.001\right]$. Thus, central capacity appears to have been shared between the two tasks, consistent with our claim that digit magnitude information in Task 2 can be retrieved in parallel with Task 1 when different tasks are performed with the stimuli for Tasks 1 and 2, if sufficient central resources are available. An additional prediction of this model that is supported by the present findings will be discussed in the following section.

\section{Locus of the Symbolic Distance Effect}

The results indicate that the symbolic distance effect was underadditive with decreasing SOA but that a residual effect of symbolic distance remained at the shortest SOAs. This finding suggests that symbolic distance affected two processing loci-one at an early stage, and one at a central (or late) stage - because the effect would have gone entirely underadditive with decreasing SOA if only an early stage had been affected or would have been entirely additive with SOA if only a central or late stage had been affected. Converging evidence in support of this claim can be found in an analysis of Task 1 RTs. When the effect of a manipulation in Task 2 has an early locus in the information-processing stream and subjects are sharing available processing capacity between the two tasks, the manipulation is predicted to have a small inverse effect on Task 1 RTs at short SOAs, but not at long SOAs, thus producing an interaction between the effect of the early Task 2 manipulation and SOA in RT1 (see Tombu \& Jolicœur, 2003, for details). That is, if symbolic distance has part of its effect at an early locus in Task 2 and processing capacity is shared between the tasks, responses to the tone should be slower with a digit that is symbolically far from the standard in Task 2 than with a digit that is symbolically close to the standard. Note that this is the opposite of what the expected effect of this manipulation would be on Task 2 RTs (faster classification of the digit when it is far from the standard than when it is near the standard). This predicted pattern of results was observed in the present experiment, although it did not quite reach significance $[F(4,424)=$ $\left.2.19, M S_{\mathrm{e}}=7,954.7, p<.07\right]$, further supporting the claim that symbolic distance has at least part of its effect at an early locus.

Which stages of processing are affected by symbolic distance? The finding of underadditivity of the effect of symbolic distance with SOA, with a residual effect at the 
shortest SOA, suggests that symbolic distance affects two processing loci, one at an early stage of processing and one at a central (or late) stage. The observed underadditivity of the symbolic distance effect could be interpreted as evidence that what was retrieved from semantic memory in parallel with the tone task in our experiment was not simply the absolute magnitude of the digit, but its classification as either larger or smaller than five (or perhaps, for some digits, as simply large or small; see below for a model based on this suggestion). This is an important distinction, since much of the evidence that digit magnitude information is retrieved autonomously has come from experiments that have shown that the quantity represented by a digit interferes with a taskrelevant aspect of the digit (e.g., its physical size; Henik \& Tzelgov, 1982). In these experiments, the absolute magnitude of the digit was a source of interference, rather than its classification along some abstract dimension (i.e., larger or smaller than five). Given that the classification of the digit in Task 2 of our experiment may have been retrieved in parallel with a central stage of processing in Task 1, the reader is left to wonder why com- plete underadditivity of the symbolic distance effect was not observed; after all, once the classification of the digit has been determined, selecting the correct response for Task 2 requires little further processing. One suggestion is that although the correct classification was retrieved in parallel with the demanding stages of Task 1, the subjects adopted a strategy of checking the retrieved classification to ensure that it was accurate. Possibly, the subjects were reluctant to act on a response that was prepotent and seemed to have been insufficiently prepared - that is, to act on a classification when there was uncertainty about where the classification came from. An interesting test of the checking hypothesis would be to repeat the experiment with children diagnosed with attention deficit hyperactivity disorder (ADHD). These children are characterized by a tendency to act on prepotent responses (see Barkley, 1997, for a review of the evidence in support of this claim). If this population bypasses a checking process and acts on the prepotent response, more underadditivity of the effect of symbolic distance with decreasing SOA would be expected than in a population without the disorder.
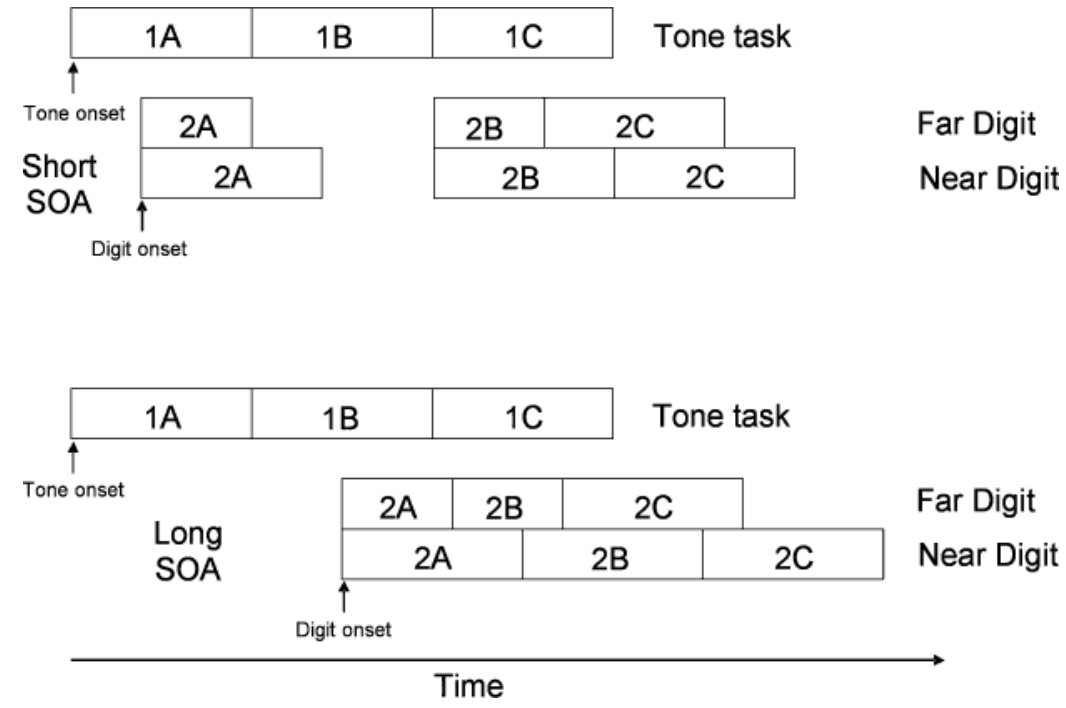

Figure 4. A model of resource requirements for the number comparison task. Each task is subdivided into three component stages. $1 \mathrm{~A}$, early stages of tone task before central resources are needed. $1 \mathrm{~B}$, central stages of tone task in which central resources are needed. $1 \mathrm{C}$, late stages of tone task after central resources are needed. $2 \mathrm{~A}$, early stages of number comparison task before central resources are needed. $2 \mathrm{~B}$, central stages of number comparison task in which central resources are needed. With a great degree of task overlap (short stimulus onset asynchrony [SOA] case, upper panel), this stage of processing is delayed until Stage $1 \mathrm{~B}$ is complete and central resources are available. With little task overlap (long-SOA case, lower panel), Stage 2B is not delayed, because Stage $1 \mathrm{~B}$ is complete by the time Stage $2 \mathrm{~B}$ is ready to begin. $2 \mathrm{C}$, late stages of number comparison task after central resources are needed. Symbolic distance affects two processing loci, one in Stage $2 \mathrm{~A}$ and one in Stage $2 \mathrm{~B}$. With an easy comparison (far digits), digits are quickly precategorized in Stage $1 \mathrm{~A}$, and response selection is accomplished quickly; as a result, the durations of both stages are short. With a difficult comparison (near digits), attempts to precategorize the digit fail, and categorization occurs at response selection; as a result, the durations of both stages are long. Although the effect of increasing the difficulty of precategorization can be absorbed into cognitive slack at a short $\mathrm{SOA}$, the effect on response selection cannot; as such, complete underadditivity is not observed. 
A second possibility is that the correct classification is retrieved in parallel with a capacity-limited stage in Task 1 but the classification affects a later stage of Task 2 (such as response selection). Consider the following model. Perhaps digits are precategorized as large or small, in parallel with Task 1 . The speed of response selection would depend, in part, on the outcome of this earlier classification stage. This situation is outlined in Figure 4. The short-SOA case is shown in the top half of the figure, whereas the long-SOA case is shown in the bottom half of the figure. The tone task in each case is decomposed into three stages (1A, 1B, and 1C). One of these stages requires central resources (1B), and two do not (1A and 1C). Similarly, the digit comparison task can be decomposed into its component stages, one requiring central resources (2B) and two that do not (2A and $2 \mathrm{C}$ ). The situation is outlined for both the case in which the digit is far from the standard (the top set of stages corresponding to Task 2) and the case in which the digit is near the standard (bottom set of stages). First, an attempt is made to classify the presented digit as large or small. Very small or large digits (e.g., 1 and 9 and, perhaps, also 2 and 8) may have a special status in that they are endpoints (or near endpoints) on the mental representation of the number line for the stimulus set used in this experiment (Banks et al., 1976; Brysbaert, 1995; Rule, 1969). Perhaps these digits are rapidly classified as greater than five or as less than five (thereby shortening Stage 2A) because they are greater than (or less than) any other digit in the stimulus set. Alternatively, these digits might become associated with large or small tags that identify the stimulus as greater than or less than five. The outcome of this classification would feed forward to response selection and shorten this stage (Stage 2B) when the stimulus has been quickly classified as small or large. This precategorization would occur in parallel with Task 1. In the event that the presented digit is close to the standard (e.g., 4, 6, 3, or 7), precategorization would succeed less frequently than when the digit is far from the standard, and a slower classification process would be carried out during response selection instead. As is shown in Figure 4, when the SOA is short (top half) and the digit is far from the standard, both Stage 2A (during which digit precategorization is assumed to be carried out) and Stage $2 \mathrm{~B}$ (during which the comparison process is assumed to be carried out) are shortened, but RT2 is affected only by the time needed for the comparison process, and not by the time needed to precategorize the digit. When the SOA is long (bottom half of Figure 4), the increase in the difficulty of both precategorizing the digit and selecting a response to the digit when it is near the standard increases RT2. Taken together, the full effect of symbolic distance is observed at long SOAs; as SOA is shortened, the effect decreases, but not entirely. This produces the observed partial underadditivity of the effect of symbolic distance with decreasing SOA. Note that this model assumes that there is sufficient postponement of Task 2 processing at short SOAs to absorb the entire effect of symbolic distance. In our experiment, decreasing SOA from 350 to
$50 \mathrm{msec}$ led to an increase in RTs of about $300 \mathrm{msec}$, suggesting that the amount of postponement was more than sufficient to absorb the entire symbolic distance effect, which was about $50 \mathrm{msec}$ at long SOAs.

The model proposed above bears some similarity to a model proposed by Banks et al. (1976) and, in fact, may be a special case of their model. Banks et al.'s model was designed to account for performance in tasks in which the standard varied from trial to trial, and both the comparison digit and the standard were presented on each trial. In their model, digits are first encoded in Stage 1 and then compared in Stage 2. Encoding generates size codes based on a crude algorithm that classifies the digits as larger or smaller than some cutoff value, which varies from trial to trial. On one trial, for example, only digits greater than 7 will be tagged with a large size code. In the subsequent comparison stage, digits precategorized as large can be quickly selected as larger than the comparison digit when the comparison digit was precategorized as small, because the two digits were tagged with different size codes at encoding (large for the larger digit, small for the smaller digit). If both of the digits were classified as large at encoding, the comparison process takes longer, because precategorization is insufficient for selecting the correct response. In our model, digits near the endpoints of the number line are precategorized quickly (as large or small, rather than larger or smaller, respectively) because the standard is fixed at five on each trial. Possibly, this model could be conceived of as a special case of Banks et al.'s (1976) model, with a fixed standard and with subjects asked to choose larger as often as they were asked to choose smaller. If so, the present work extends their model by suggesting that the encoding stage responsible for quickly generating size codes does not require central resources, whereas the comparison stage that follows does require central resources.

The model proposed above also shares features with a more general model of dual-task performance described by Hommel (1998), but it is different in several important ways. Hommel argued that responses in Task 2 can be activated in parallel with resource-demanding stages of Task 1 but response selection in Task 2 must wait until resource-demanding stages of Task 1 are complete. According to Hommel's model, stimuli are automatically translated into their responses in parallel, with final selection of a response carried out in a later, controlled stage. The automatic translation of stimuli in Task 2 into their responses might be viewed as analogous to the precategorization stage in our model, and the final, controlled selection of a response might be viewed as analogous to the comparison stage in our model. It is important to note, however, that in our model, the stage of precategorization in Task 2 is carried out only when initial analysis determines that the digit is far from the standard. In that sense, our model differs from Hommel's, because his model would predict that stimuli are always translated into their responses automatically. In addition, in our model, the response (less than five or more than five) in Task 2 is not 
automatically activated at the time the digit is presented; rather, we argue that a gross precategorization of the digit as large or small takes place.

Our two-stage model argues that, when the digit is close to the standard (near 5), classifying the digit as less or more than five requires a resource-demanding comparison stage. If so, the classification of the digit (more than five or less than five) should be less available at the time at which the pitch of the tone is classified than when the digit is far from the standard. Thus, the model predicts that tone frequency and digit classification will influence each other more when the digit is far from the standard (and is grossly precategorized as high or low) than when it is near the standard (and cannot be precategorized in this way), and in fact, this is exactly what the results of the present experiment suggest. That is, the effect of the digit classification on tone responses was larger with far digits than with near digits, and the effect of tone classification on digit responses was larger with far digits than with near digits.

An interesting broader theoretical implication of the results of the present study is that categorizing a stimulus in an absolute manner (e.g., as large or small) can be done without requiring limited-capacity attentional resources but that categorizing this same stimulus relative to a standard cannot. Future work should be directed at testing some of the predictions generated by this broader theoretical framework. For example, repeating the present experiment with stimuli that are readily categorized as small or large (e.g., the stimuli near the endpoints in the range of stimuli used in this experiment) should produce complete underadditivity of the effect of symbolic distance with decreasing SOA, because the resourcedemanding stage of comparing the digit with a standard would be eliminated.

In summary, the present work suggests that, in contrast to Logan and Schulkind's (2000) results, information relevant to digit magnitude classification in Task 2 was retrieved in parallel with Task 1, despite the fact that the task sets for these tasks were different. The important variable appears to be whether the task sets for the two tasks can be held concurrently; when they cannot, parallel retrieval might not be possible. The results of our experiment suggest that comparing a presented digit with a fixed internal standard involves both a stage of processing that can be carried out while central resources are allocated to another task and a stage of processing that cannot be carried out until the stages of Task 1 requiring central resources are complete.

\section{REFERENCES}

Allport, A., Styles, E. A., \& Hsieh, S. (1994). Shifting intentional set: Exploring the dynamic control of tasks. In C. Umiltà \& M. Moscovitch (Eds.), Attention and performance XV: Conscious and nonconscious information processing (pp. 421-452). Cambridge, MA: MIT Press, Bradford Books.

Banks, W. P., Fumil, M., \& Kayra-Stuart, F. (1976). Semantic congruity effects in comparative judgments of magnitudes of digits. Journal of Experimental Psychology: Human Perception \& Performance, 2, 435447.
BARKLEY, R. (1997). ADHD and the nature of self-control. New York: Guilford.

BRysbaert, M. (1995). Arabic number reading: On the nature of the numerical scale and the origin of phonological recoding. Journal of Experimental Psychology: General, 124, 434-452.

CARrier, L. M., \& PAShler, H. (1995). Attentional limits in memory retrieval. Journal of Experimental Psychology: Learning, Memory, \& Cognition, 21, 1339-1348.

Dehaene, S., \& Akhavein, R. (1995). Attention, automaticity, and levels of representation in number processing. Journal of Experimental Psychology: Learning, Memory, \& Cognition, 21, 314-326.

Dehaene, S., Bossini, S., \& Giraux, P. (1993). The mental representation of parity and number magnitude. Journal of Experimental Psychology: General, 122, 371-396.

Fox, L. A., Shor, R. E., \& Steinman, R. J. (1971). Semantic gradients and interference in naming color, spatial direction, and numerosity. Journal of Experimental Psychology, 91, 59-65.

HeniK, A., \& Tzelgov, J. (1982). Is three greater than five: The relation between physical and semantic size in comparison tasks. Memory \& Cognition, 10, 389-395.

Hommel, B. (1998). Automatic stimulus-response translation in dualtask performance. Journal of Experimental Psychology: Human Perception \& Performance, 24, 1368-1384.

JersiLd, A. T. (1927). Mental set and shift. Archives of Psychology, 89, $5-82$.

Logan, G. D., \& SchulKind, M. D. (2000). Parallel memory retrieval in dual-task situations: I. Semantic memory. Journal of Experimental Psychology: Human Perception \& Performance, 26, 1072-1090.

McCann, R. S., \& Johnston, J. C. (1992). Locus of the single-channel bottleneck in dual-task interference. Journal of Experimental Psychology: Human Perception \& Performance, 18, 471-484.

MoYer, R. S., \& LANDAUER, T. K. (1967). Time required for judgments of numerical inequality. Nature, 215, 1519-1520.

NAVON, D., \& Miller, J. (2002). Queuing or sharing? A critical evaluation of the single-bottleneck notion. Cognitive Psychology, 44, 193251.

Oriet, C., \& Jolicceur, P. (2003). Absence of perceptual processing during reconfiguration of task set. Journal of Experimental Psychology: Human Perception \& Performance, 29, 1036-1049.

Pashler, H. (1994). Dual-task interference in simple tasks: Data and theory. Psychological Bulletin, 116, 220-244.

Pashler, H., \& Johnston, J. C. (1989). Chronometric evidence for central postponement in temporally overlapping tasks. Quarterly Journal of Experimental Psychology, 41A,19-45.

Rogers, R. D., \& Monsell, S. (1995). Costs of a predictable switch between simple cognitive tasks. Journal of Experimental Psychology: General, 124, 207-231.

RULE, S. (1969). Equal discriminability scale of number. Journal of Experimental Psychology, 79, 35-38.

Spector, A., \& Biederman, I. (1976). Mental set and mental shift revisited. American Journal of Psychology, 89, 669-679.

Sudevan, P., \& TAYLOR, D. A. (1987). The cuing and priming of cognitive operations. Journal of Experimental Psychology: Human Perception \& Performance, 13, 89-103.

Tombu, M., \& Joliceur, P. (2003). A central capacity sharing model of dual-task performance. Journal of Experimental Psychology: Human Perception \& Performance, 29, 3-18.

Tzelgov, J., Meyer, J., \& HeniK, A. (1992). Automatic and intentional processing of numerical information. Journal of Experimental Psychology: Learning, Memory, \& Cognition, 18, 166-179.

VAN SelST, M., \& Joliceur, P. (1994). A solution to the effect of sample size on outlier elimination. Quarterly Journal of Experimental Psychology, 47A, 631-650.

Van Selst, M., \& Jolicceur, P. (1997). Decision and response in dualtask interference. Cognitive Psychology, 33, 266-307.

WINDES, J. (1968). Reaction time for numerical coding and naming of numerals. Journal of Experimental Psychology, 78, 318-322.

(Manuscript received December 6, 2002; revision accepted for publication August 14, 2004.) 